\title{
Present Scenario and Prospect of Model Pharmacy in Dhaka City, Bangladesh
}

\section{Md. Hasinur Rahaman Habib ${ }^{1}$, Nushrat Alam ${ }^{1}$, Mimosa Kamal' ${ }^{2}$, Muhammad Rashedul Islam ${ }^{2}$ and Md. Elias Al-Mamun ${ }^{2}$}

${ }^{1}$ Department of Pharmacy, Faculty of Pharmacy, University of Dhaka, Dhaka-1000, Bangladesh

${ }^{2}$ Department of Pharmaceutical Technology, Faculty of Pharmacy, University of Dhaka

Dhaka-1000, Bangladesh

(Received: June 10, 2020; Accepted: July 19, 2020; Published (web): July 25, 2020)

\begin{abstract}
The pharmaceutical sector is one of the most promising sectors of Bangladesh. With a view to elimitate the improper dispensing practice as well as matching up to the advancing global standard of health care system, the Ministry of Health and Family Welfare (MHFW), Government of the People's Republic of Bangladesh had launched the "Model Pharmacy" program in 2016. Our aim was to find out the perception of general people regarding model pharmacy and the study the overall impact in our healthcare system due to its inauguration. A survey was conducted among the major stakeholders of model pharmacy system such as patients, physicians, retailers and DGDA personnel and the data were correlated. All the stakeholders applauded the initiative. However, each section pointed out specific problems. According to the retailers, flexibility in the license getting process was a must as they found the process to be lengthy $(31.43 \%)$, unnecessarily strict $(22.86 \%)$ and corrupted $(22.86 \%)$. The physicians felt the program needed more exposure and recommended campaigning (23.33\%). Additionally, they recommended to build up databases of doctors $(46.67 \%)$ and model pharmacies (50\%) for the ease of collaboration between them and the retail pharmacists. Patients were in favor of introducing more model pharmacies (20\%) and recruitment of A-grade Pharmacists (6.67\%). DGDA personnel noticed the miscommunication between physicians, retail pharmacists and authority to be a major problem. All the stakeholders unanimously focused on regular monitoring of the program to make it a success. Beyond this discussion, we are hopeful that with adequate measures and continuous modification, the "Model Pharmacy" program would become successful in Bangladesh.
\end{abstract}

Keywords: Model pharmacy, Patient, Physician, Retail pharmacist.

\section{Introduction}

Pharmaceutical sector has become one of the most promising sectors in the recent years in Bangladesh (Sultana, 2016). However, due to improper management of drugs, sale of adulterated and substandard drugs as well as the lack of patient counselling, the sector has not been fully developed yet (Elias et al., 2016). Retail pharmacies are the market place from where the patients purchase their medicines as per their need (Sultana, 2018). There has been a worldwide shift in the role of community pharmacies in the healthcare system where the simple medication dispenser has now been promoted to a healthcare provider (Mossialos et al., 2015). Unfortunately, in Bangladesh the conventional retail pharmacies are still under the control of personnel with C-grade registration from the Pharmacy Council of Bangladesh. In majority of the cases, the owners of many pharmacies fail to employ at least one diploma pharmacist or pharmacy technician, which is a requirement for setting up a pharmacy. With such a setup and inadequate efforts from the respective authority, we are now facing problems that may be fatal enough to human health such as emergence of 
drug resistance, sale of substandard and counterfeit drugs, lack of proper patient-counseling, pricing problem, improper storage of drugs, unhygienic dispensing of drug, absence of record keeping and documentation system, improper disposal of damaged or expired medicines and so on.

There has been tremendous progress in our health sector in the recent years. To eradicate the current improper dispensing system along with a view to improving the accessibility, quality and affordability of health care to meet the needs of our population, the Ministry of Health and Family Welfare (MHFW) had promulgated The National Drug Policy in 2016 and introduced 30 Model Pharmacies on 22nd December, 2016 as a pilot program (Sultana, 2018).

A Model Pharmacy is a community-based pharmacy practice, where the responsibilities of pharmacists include checking and dispensing of prescribed drugs, providing advice on drug selection and usage to doctors and other health professionals and counseling patients on health promotion, disease prevention and the proper use of medicines. This program does not only ensure the betterment of healthcare system but also creates new job opportunities for graduate pharmacists. In this age, the retail pharmacists or A-grade pharmacists play important roles in medicine management as well as bring changes in the pattern of the rapid growth of domestic medicine use and national healthcare expenditure (Dalton and Byrne, 2017). The sole purpose behind introducing this program was to control drug dispensing system with specific requirements for storage conditions, equipment and record keeping as well as stop fake drug sales in Bangladesh.

Starting from 7th March, 2018, the Directorate General of Drug Administration (DGDA) under the MHFW has introduced 193 model pharmacies in Bangladesh and the majority of them are situated in Dhaka city (web-1). Compared to the requirements, the number of model pharmacies is very limited and the existing ones have too many shortcomings such as the lack of A-grade Pharmacists and properly trained health personnel, underdeveloped setup that prevent the retail pharmacists from drug compounding, lack of communication between different groups of health professionals specially between retail pharmacists and physicians and so on. That is why we have tried to find out the present scenario of model pharmacies in Bangladesh and gathered the perception of general people and physicians about model pharmacies as well as the resulting changes in the overall healthcare system before pursuing the authority to expand this program in different districts and upazilas of Bangladesh.

\section{Materials and Method}

Design of experiment: We reviewed necessary literature and existing similar systems in different countries. Depending on the data gathered from literature review, we decided to carry out the research (Figure 1). The next step for this project was data collection. In this step, we collected the data from DGDA personnel, retail pharmacists, patients and physicians as they are the main stakeholders of model pharmacy. Then these data were analyzed to get information about response of all the stakeholders towards model pharmacy, shortcomings of the system and what could be the future plan to solve these issues.

Selection of survey area: We selected Dhaka for our proposed survey work, as the city being the capital of Bangladesh, would be an appropriate place to get a brief scenario of model pharmacy in our country. Besides the DGDA office and many tertiary level hospitals existing here could help us to get survey related information.

Selection of stakeholders: The respondents of this study were patients, physicians, retailers and the concerned officers of DGDA. We selected patients and physicians from different medical college hospitals. We interviewed the retail pharmacists who were working in the recently established model pharmacies and visited DGDA office to interview the officers.

Data sources: We selected two medical college hospitals for our research work that included - Dhaka 
Medical College Hospital and Sir Salimullah Medical College Hospital. Doctors of these medical colleges helped us to get the necessary information. But only patients of Dhaka Medical College Hospital were involved in our survey work. We visited many model pharmacies situated at different parts of Dhaka city, such as- Shahbagh, Kalabagan, Mohammadpur, Banani, and Uttara to get the necessary information. Besides, in DGDA five Superintendent of Drug helped us to gather necessary information.

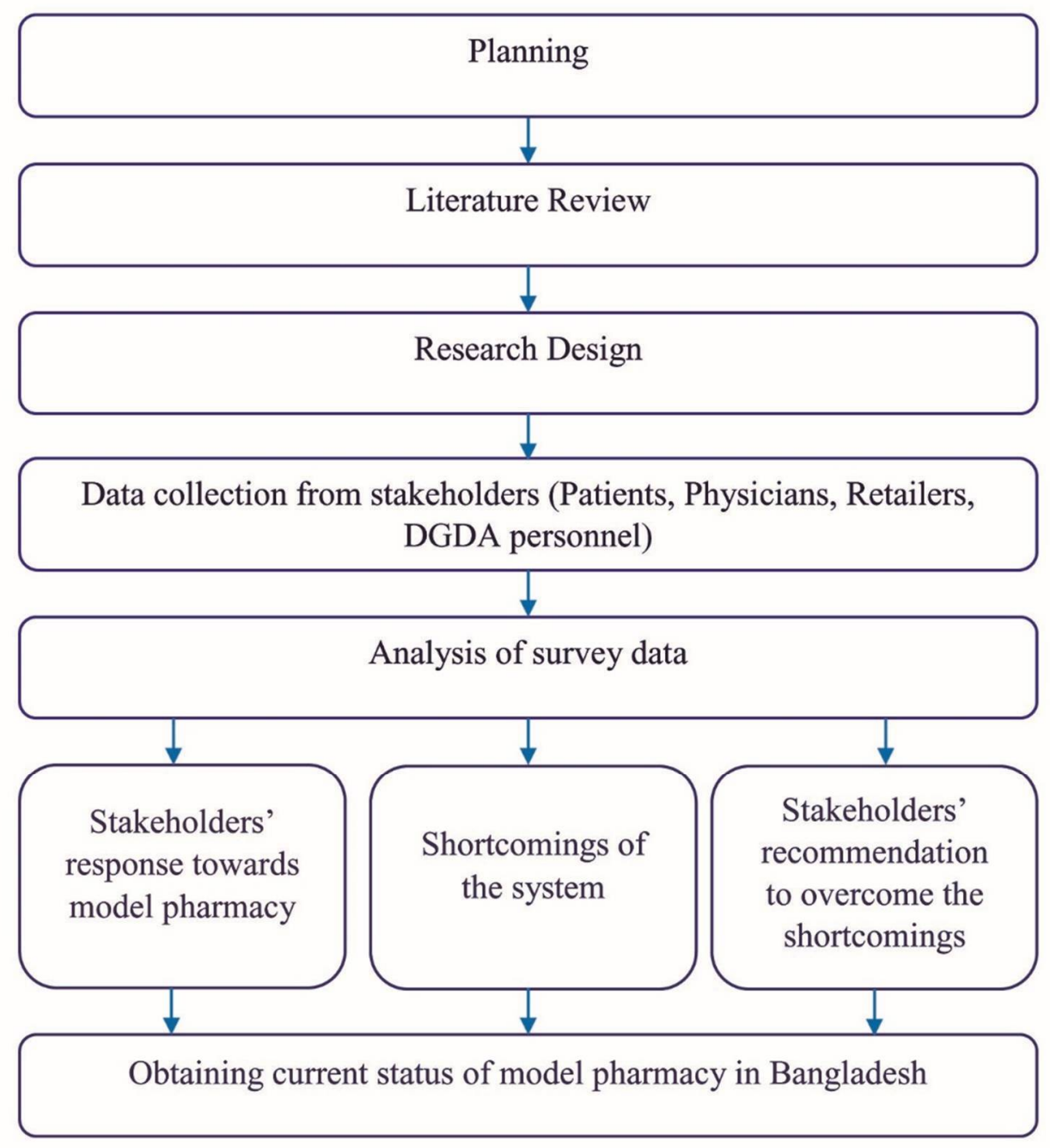

Figure 1. Methodology flowchart

Data collection: Required information about the study area was obtained by preliminary field investigation. The perception of the respondents and suggestions were taken from the selected medical colleges and also from the respondents of model pharmacies and DGDA office. 100 survey sheet were prepared. Among these, 35 were prepared for retailers, 30 for patients, 30 for physicians and 5 for DGDA officers.
Data analysis: The data collected from our studies were processed and analyzed by using both descriptive and inferential statistical data by using MS Word and MS Excel.

\section{Results and Discussion}

Purpose of initiating model pharmacy system: According to the DGDA personnel, the government has initiated the model pharmacy system solely for 
improvement of the current system of dispensing medicines that would benefit the general people.

Number of current licensed pharmacies and model pharmacies: Currently there are approximately $1,25,000$ pharmacies established in Bangladesh. Among them only 193 pharmacies are model pharmacy, most of which were licensed in 2017 by DGDA (web-1). We received similar data from our respondent retailers as 32 out of 35 of them got their license in 2017.

Reasons behind not switching to model pharmacy from regular pharmacy: There are several reasons behind this attitude of not switching to model pharmacy. The major problem stated by the DGDA personnel is cost since recruitment of A-grade pharmacists and installation of better facilities - both are essential but very expensive.

Comments regarding the process of getting license from DGDA: According to the DGDA personnel, model pharmacy owners are satisfied with the registration procedure of model pharmacy. However, we observed the pharmacy owners were quite dissatisfied with the current registration procedure. About $31.43 \%$ retailers found the process to be very lengthy, $22.86 \%$ retailers opined that the license getting process is overly strict, $22.86 \%$ retailers felt that sometimes corruptions or misdeeds were involved to get the registration or licenses that were shown in figure 2 . The remaining $22.86 \%$ retailers thought that the process was quite good (Figure 2).

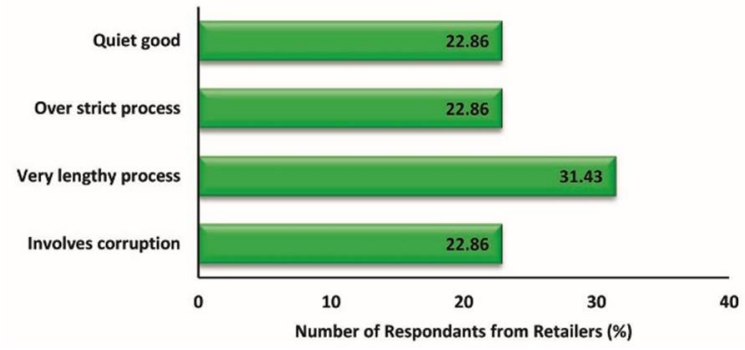

Figure 2. Comment of retailers about license getting process.

Storage hard copies of prescriptions and record keeping: Among the 35 model pharmacy we visited, $34.29 \%$ of them indicated that they store hard copies of the prescriptions for one year. The remaining
$65.71 \%$ of the model pharmacies were found to be poor in record keeping and they didn't store any prescription they served.

Data base system in model pharmacy: All the model pharmacy included in our survey had good data base system.

Perception about model pharmacy and resulting change in overall health system: About $73.33 \%$ patients were aware about model pharmacy and it affected their buying behavior. On the other hand, $53.33 \%$ of the responding patients started buying medicines from the model pharmacies (Figure 3). However, irrespective of their awareness, $16.67 \%$ patients continued to buy medicines from their local pharmacies, $6.67 \%$ patients bought medicines from the hospital pharmacies and the remaining $23.33 \%$ patients preferred to buy medicines from pharmacies that were located near the physicians' chambers as they thought the quality of the medicines would be better.

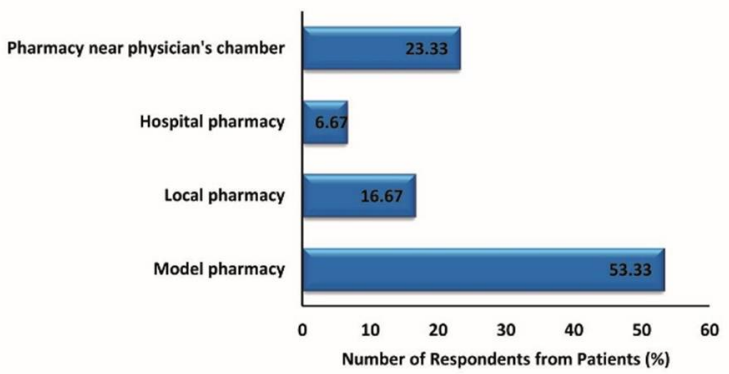

Figure 3. Preference of purchasing medicines by patients from different pharmacies.

Similarly, we received positive responses from the physicians. All of the physicians involved in our project work believed that patients would get more benefits from model pharmacy and $90 \%$ of them opined that collaboration between doctors and pharmacists would be beneficial for our health sector.

However, in reality only $30 \%$ physicians said that retailers had consulted with them about prescription related problems. That is why 50\% physicians opined that the introduction of model pharmacy has been a very good initiative while $26.67 \%$ physician said the rules and regulations have to be maintained strictly during running the model 
pharmacies and the remaining $23.33 \%$ physician emphasized on more campaigning on model pharmacy to increase awareness among patients and retailers (Figure 4).

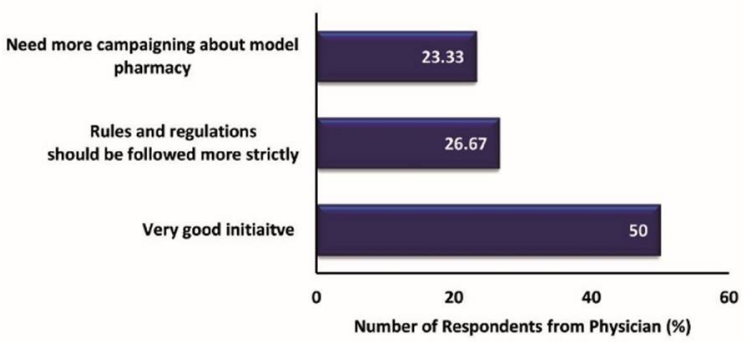

Figure 4. Perception of the Physicians regarding model pharmacy.

Actions taken by patients when they could not find the prescribed brands of medicines: Among the patients, $66.67 \%$ changed the prescribed brands of their medicines as per the suggestion of the retailers, $6.67 \%$ patients changed the brands by themselves, $10 \%$ patients consulted with the pharmacists while the remaining $16.67 \%$ patients consulted with their doctors (Figure 5).

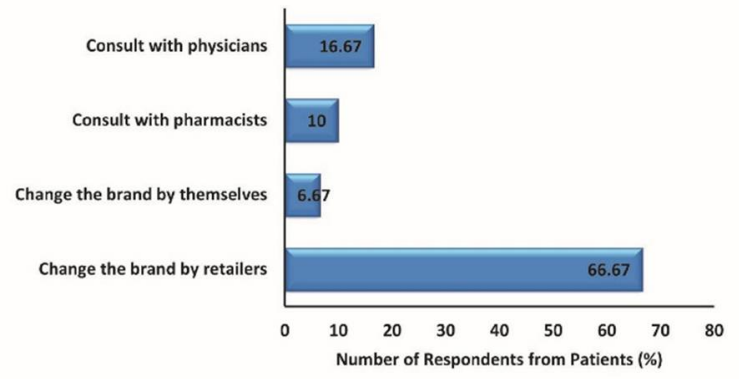

Figure 5. Action taken by patients when prescribed brand of medicines could not be found.
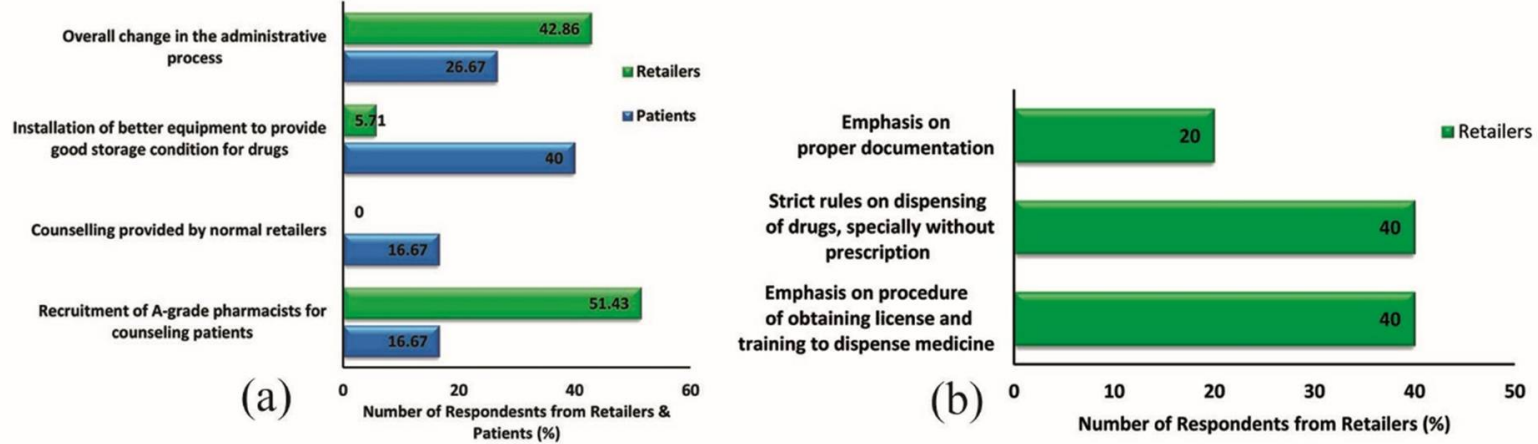

Figure 6 (a). Common changes observed by both patients and retailers in model pharmacy. (b). Changes observed by retailers in the administrative process in model pharmacy.
Changes observed in model pharmacy over conventional retail pharmacy: According to the opinions of $16.67 \%$ patients, recruitment of A-grade pharmacist for patient counseling has been the major advantage of a model pharmacy (Figure 6a). However, equal portion of patients said that they had received counselling from normal retailers and majority of the patients (40\%) pointed towards good storage condition for drugs in model pharmacy. About $26.67 \%$ patients agreed that some changes have been visible in the overall administration process due to the introduction of model pharmacies but it didn't reflect much on the dispensing procedure. On the contrary, the majority of the retailers $(51.43 \%)$ thought that the recruitment of Agrade pharmacist has been the major change. None of the respondents talked about counselling provided by the normal retailers. About $5.71 \%$ retailers mentioned about the installation of better equipment to provide good storage condition for drugs. However, another major portion $(42.86 \%)$ of the retailers gave emphasis on the changes observed in the administrative process.

Among the retailers, $40 \%$ retailers put emphasis about the procedure of obtaining license and training to dispense medicines, $40 \%$ retailers felt rules for dispensing of drug have become more stringent, especially those without prescription (Figure 6b). The remaining $20 \%$ retailers focused on proper documentation in the model pharmacies. 
Model pharmacy monitoring system: Generally, the field level officers of DGDA monitor the model pharmacies and they do it regularly. However, according to the officials of DGDA, most of the retailers are reluctant to follow the rules and regulations properly.

Recommendations about the improvement of model pharmacy: When we asked responding patients, physicians and retailers about their suggestions for the improvement of model pharmacy, $46.60 \%$ patients, $36.67 \%$ physicians and $36.67 \%$ retailers put emphasis on regular monitoring of model pharmacy (Figure 7a). However, each stakeholder had some specific recommendation that would help to improve the existing system. About $6.67 \%$ patients said more A-grade pharmacists should be recruited whereas $20 \%$ patients suggested to increase the number of model pharmacies (Figure $7 \mathrm{~b}$ ). The remaining $26.67 \%$ patients gave no suggestions. Our respondent physicians specifically focused on proper communication between physicians and A-grade pharmacists. As we observed, apart from focusing on the monitoring system, $46.67 \%$ of the total physicians suggested to build database of doctors so that pharmacists working in model pharmacies could easily reach them (Figure 7c). The physicians (40\%) included in our studies, opined that the model pharmacies should give more emphasis on good data base system. Our respondent retailers felt that the existing rules and regulations should be improved. Individually $46.67 \%$ retailers wanted the existing licensing procedure should be less time consuming, whereas $26.67 \%$ retailers thought that the rules and regulations for guiding the model pharmacy should be more flexible (Figure 7d). Remaining $6.67 \%$ retailers said that the DGDA should ensure that the retailers can get help with necessary information promptly.

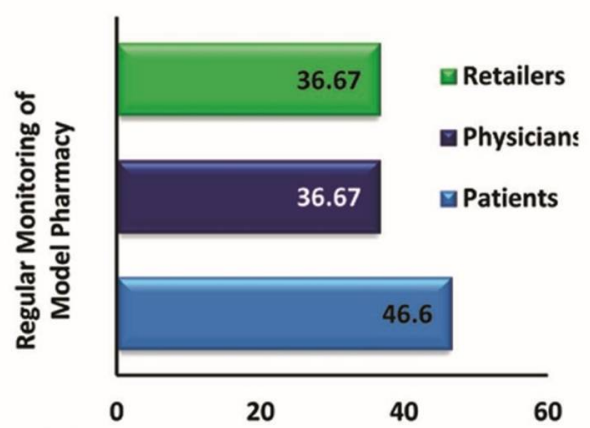

(a) Number of Respondents from all Stakeholders (\%)

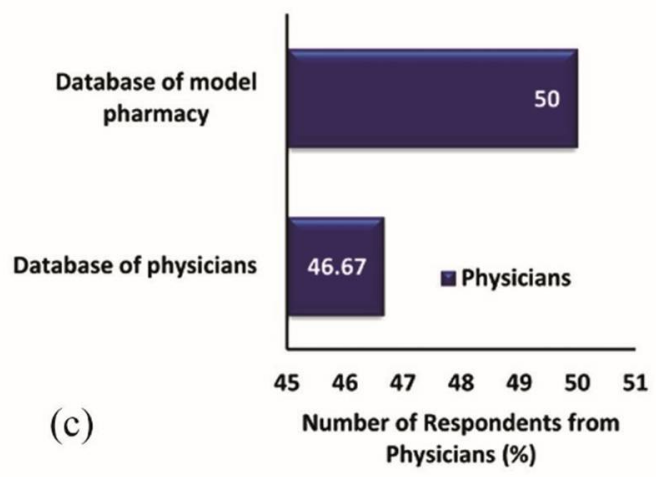

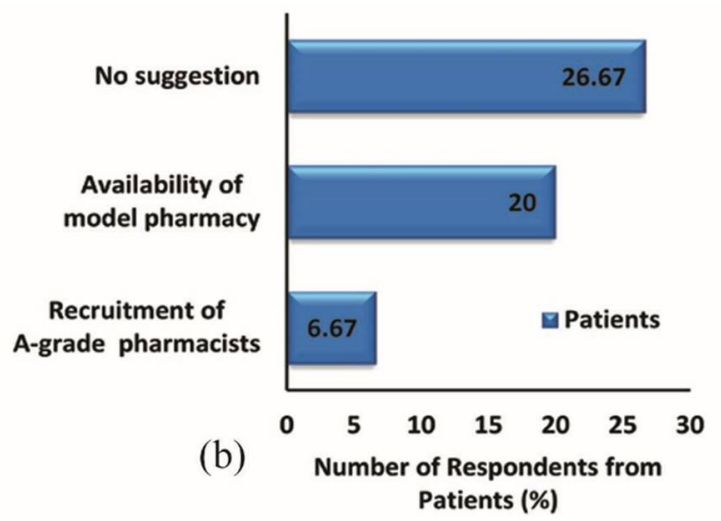

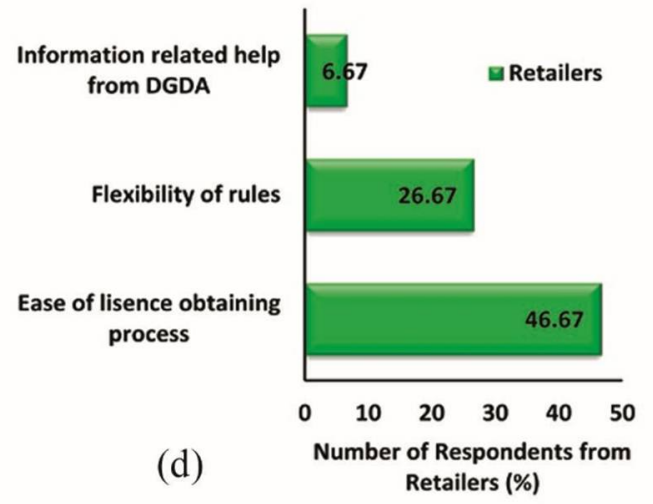

Figure 7(a) Common recommendation by all the stakeholders for model pharmacy. (b) Recommendation by the patients for model pharmacy. (c) Recommendation by the physicians for model pharmacy. (d) Recommendation by the retailers for model pharmacy. 
This study about the present scenario of model pharmacy in Bangladesh was conducted among patients, physicians, retailers and DGDA personnel. It enabled us to understand the perception of general people regarding the model pharmacy, the change this system has brought into our healthcare system, limitations associated with model pharmacies and recommendations of patients as well as associated physicians and retailers which could help improve this program further.

The main purpose of initiating the model pharmacy was to ensure health benefits of general people by appointing A-grade pharmacists in community pharmacies. Since the program was initiated in December 2016, the number of approved model pharmacies was very low during the first year. The number increased over the following years as a large number of pharmacy related businessmen got attracted to this program due to the campaigning across the country and took permission from DGDA to run model pharmacy. However, we observed a decreasing pattern in obtaining the license for running a model pharmacy in the year 2018 compared to previous year. This was consistent with the data we obtained from the retailers as 32 out of 35 got their license in 2017 while in 2018, only 2 of them got their license. On the question of what might be the reason behind this negative trend, the DGDA officials referred to the cost being the prime reason as recruitment of A-grade pharmacists and installation of better facilities are really expensive. As a result, majority of the pharmacy owners failed to obtain their license and convert the retail pharmacies into model pharmacies. Hence, the pharmacy owners think that the government should give them financial support, such as easy bank loan. Other than that, we observed communication gap between retailers and the DGDA personnel. The DGDA staffs feel that the model pharmacy owners are satisfied with the current registration procedure of model pharmacy while in reality, only $22.86 \%$ retailers indicated their satisfaction with the existing system. The remaining $77.14 \%$ retailers pointed out to many problems such as - corruption, long time to get the license and over stringent nature of the process (Figure 2).

However, these findings were limited to the retailers and governing authorities. The perceptions of the patients and physicians regarding the model pharmacy was also required to get a clear idea about the limitations of model pharmacy and the extent of positive change that this newly initiated program has brought into our existing healthcare system. Fortunately, $73.33 \%$ patients were aware of model pharmacy. Considering model pharmacy program was launched in December 2016, the number was quite high. However, only $53.33 \%$ of these patients bought medicine from model pharmacy. Inadequate number of model pharmacy as well as retailers' influence on patients' buying behavior was the major reason behind the patients opting for local pharmacy despite their awareness. This was consistent with the finding that if a certain medicine was out of stock, $66.67 \%$ patients changed the brand of their medicine solely based on the retailers' suggestion (Figure 5).

We obtained positive response from the physicians too. Collaboration between pharmacists and doctors in health related issues is important for modernization of health sector (Seselja-Persin et. al., 2016) and $96.67 \%$ of the responding doctors stressed on the development of separate databases containing list of the physicians $(46.67 \%)$ and model pharmacies $(50 \%)$ so that both the professionals could seek professional help from each other (Figure 7c). Unfortunately, we observed a huge lack of communication between the physicians and retailers. All the model pharmacy included in our survey had proper database and $34.29 \%$ of them stored hard copies of prescription for a year. About 23.33\% physician emphasized on more campaigning activity so that all the stakeholders including the patients, retailers and physicians get a clear idea about model pharmacy and their responsibilities. This will also help to follow the rules properly thereby improve the present situation.

On the question of the changes that model pharmacy program has resulted in, both patients and retailers pointed to the recruitment of A-grade 
pharmacists and installation of better instruments for ensuring the quality of drugs (Figure 6a). However, patients pointed on counselling provided by A-grade pharmacists, which wasn't followed earlier. This observation was consistent with the claim of the Pharmaceutical Society of Singapore who stressed on pharmacists' role in team-based care in making decision as well as allow the physicians to focus on diagnosis and treatment (web-2). In accordance, the other changes noticed by the retailers was the change in administrative process, the change in proper documentation and dispensing.

\section{Recommendations}

Patients in our country suffer a lot due to improper patient counseling and mismanagement of drugs. The lack of proper storage condition and digitalized system further adds to the problem. Model pharmacy program was introduced with a view to solving these problems. However, the program is still in its initial phase and lots of modifications and adjustments are needed to be done for achieving the ultimate goal. Based on our survey, we recommend that the recruitment of adequate number of A-grade and C-grade pharmacists is a must in a model pharmacy. Each model pharmacy should have a separate area where an A-grade pharmacist will perform counseling with the patients for their prescribed medicines. A digitalized system should also be maintained for keeping records of patients and medicines. Introduction of online drug delivery system under the model pharmacy could further improve patient's satisfaction. The purpose of establishing model pharmacy wouldn't be fulfilled unless a proper communication is established between pharmacists and physicians. This could be the beginning of the development of a Diseases and Diagnostics Database (DDD) system which would help to develop our health sector as our study group proposed previously (Elias et al., 2016). It is essential to gradually scale up and disseminate model pharmacy program in rural areas to ensure better health quality of general people in those areas. Regular monitoring of model pharmacy by DGDA staffs would further ensure the success of the program. Time bound and updated training program should be offered to the DGDA staffs to increase their efficiency.

\section{Conclusion}

From our study, we found that the model pharmacy in Bangladesh is still at the primary stage. There is still a lack of knowledge among the retail pharmacists regarding safety of medicine, usage and storage, which has a profound impact on the outcomes of therapy. This is partly due to lake of proper education and training for the retail pharmacists. Therefore, to ensure the safe and proper dispensing of the medications, the pharmacist should be properly trained and educated. Besides, easy license obtaining procedures as well as adequate financial incentives should be made available by DGDA and collaboration between pharmacists and physicians must be ensured to provide better health services for general people. Finally, we can say that if all the stakeholders could follow their recommendations mentioned here, our people would be able to get proper medical services from model pharmacies like the developed countries.

\section{References}

Dalton, K. and Byrne, S. 2017. Role of the pharmacist in reducing healthcare costs: current insights. Integrated Pharm. Res. Pract. 6, 37-46.

Elias-Al-Mamun, M., Saha,T. and Islam, M.R. 2016. Initiation and Maintenance of Diseases and Diagnostic Databases of Bangladeshi Individual. American $J$. Pharm. Health Res. 4, 30-42.

Mossialos, E., Courtin, E., Naci, H., Benrimoj, S., Bouvy, M., Farris, K., Noyce, P. and Sketris, I. 2015. From "retailers" to health care providers: transforming the role of community pharmacists in chronic disease management. Health Policy. 119, 628-639.

Seselja-Perisin, A., Mestrovic, A., Klinar, I. and Modun, D. 2015. Health care professionals' and students' attitude toward collaboration between pharmacists and physicians in Croatia. Intl. J. Clin. Pharm. 38, 16-19.

Sultana, J. 2016. Future Prospects and Barriers of Pharmaceutical Industries in Bangladesh. Bangladesh Pharm. J. 19, 53-57. 
Sultana, J. 2018. Patients' perception and satisfaction on model pharmacies in Dhaka city, Bangladesh. Bangladesh Pharm. JA. 21, 47-54.

Web-1: Model pharmacy guidelines and list.

http://www.dgda.gov.bd/index.php/pharmacies/whole-salepharmacy-view-2 (Accessed on: 5-7-2020)
Web-2: Pharmacists play vital role in medication counselling

https://www.straitstimes.com/forum/letters-inprint/pharmacists-play-vital-role-in-medicationcounselling (Accessed on: 6-7-2020) 